\title{
Imaging spin diffusion in germanium at room temperature
}

\author{
C. Zucchetti, ${ }^{1}$ C. Vergnaud,${ }^{2}$ F. Bottegoni,${ }^{1}$ F. Ciccacci,${ }^{1}$ G. Isella,${ }^{1}$ L. Ghirardini, ${ }^{1}$ \\ M. Celebrano, ${ }^{1}$ F. Rortais, ${ }^{2}$ A. Ferrari, ${ }^{2}$ A. Marty, ${ }^{2}$ M. Finazzi,${ }^{1,}{ }^{*}$ and M. Jamet ${ }^{2}$ \\ ${ }^{1}$ LNESS-Dipartimento di Fisica, Politecnico di Milano, 20133 Milano, Italy \\ ${ }^{2}$ Spintec, Institut Nanosciences et Cryogénie, Univ. Grenoble Alpes, CEA, CNRS, F-38000 Grenoble, France
}

(Dated: February 28, 2017)

\begin{abstract}
We report on the nonlocal detection of optically-oriented spins in $n$-type germanium at room temperature. Localized spin generation is achieved by scanning a circularly polarized laser beam $(\lambda=1550 \mathrm{~nm})$ on an array of lithographically defined Pt microstructures. The in-plane oriented spin generated at the edges of such microstuctures, placed at different distances from a spin-detection element, allows for a direct imaging of spin diffusion in the semiconductor. Two different spindetection blocks are employed, consisting either in a magnetic tunnel junction or a platinum stripe where the inverse spin-Hall effect converts the spin current into an electrical signal. The second solution represents the realization of a nonlocal spin injection/detection scheme that is completely free from ferromagnetic functional blocks. We could finally extract a spin diffusion length in lightly doped germanium as long as $10 \mu \mathrm{m}$ at room temperature.

PACS numbers: 72.25.Dc, 72.25.Fe, 72.25.Rb, 72.25.Mk, 85.75.-d
\end{abstract}

Spintronics aims at exploiting the spin degree of freedom to manipulate information, which, in conventional electronics, is instead associated only with the charge of carriers [1-3]. In this regard, germanium appears as a promising hosting material for spin transport and manipulation. The electron spin lifetime can reach several nanoseconds at room temperature [4] and the compatibility with mainstream silicon technology allows exploiting the spin-related properties of low dimensional SiGeheterostructures $[5,6]$.

Moreover, selection rules for the absorption of circularly polarized light at the direct gap of Ge, which perfectly matches the $1550 \mathrm{~nm}$ telecom wavelength, enable spin injection by means of optical spin orientation [7]. Once optically excited at the $\Gamma$ point, electrons are scattered to the L valleys of the Brillouin zone still maintaining, at least partially, their spin polarization [8-10]. The long lived-L states can then be used for spin transport and detection [11-13].

Electrical spin injection and detection has been explored in Ge films or nanowires using either nonlocal measurements in lateral or vertical spin valves [14-17] or the Hanle effect in three-terminal devices [18-25]. The nonlocal lateral geometry is particularly interesting in spintronics since it allows, in principle, spin manipulation in the channel between the spin injector and detector. However, experimental measurements based on magnetic tunnel junctions (MTJ) have been limited in temperature to $225 \mathrm{~K}$ [14] and the only demonstration at room temperature was performed indirectly with a method combining spin pumping and inverse spin Hall effect (ISHE) [26].

Here we implement a novel nonlocal spin injection/detection scheme in germanium at room temperature, adding new functionalities to the common architectures available for spintronic devices. By exploiting optical spin generation and a set of lithographically de- fined metal microstructures, we demonstrate lateral spin transport in a bulk lightly $n$-doped Ge sample. Nonlocal spin detection is achieved using either a MTJ or the ISHE in a Pt bar. With this setup we directly show optical mapping of spin diffusion in Ge and, by combining optical spin orientation and the ISHE in Pt, we build a nonlocal spin injection/detection scheme without the use of any ferromagnetic metal. We find a spin diffusion length of about $10 \mu \mathrm{m}$ at room temperature making germanium an ideal platform for fundamental and applied research in spintronics.

A $\mathrm{Ge}(001)$ substrate $n$-doped with As $\left(n=1.7 \times 10^{16} \mathrm{~cm}^{-3}\right)$ was first cleaned in acetone and isopropyl alcohol into an ultrasonic bath for $5 \mathrm{~min}$ and then rinsed into deionized water before being loaded into the MBE chamber. Successively, the native Ge oxide was thermally removed by annealing under ultrahigh vacuum to obtain a well-defined $(2 \times 1)$ surface reconstruction, as detected by reflection high-energy electron diffraction. For the MTJ device (see Fig. 1a), we first deposited a $8 \mathrm{~nm}$-thick $\mathrm{MgO}$ layer at $310^{\circ} \mathrm{C}$, followed by a $10 \mathrm{~min}$ annealing at $650{ }^{\circ} \mathrm{C}$ and by room-temperature deposition of $15 \mathrm{~nm}$ of Pt. Eight $1 \times 2 \mu \mathrm{m}^{2} \mathrm{Pt} / \mathrm{MgO}$ pads separated by $1 \mu \mathrm{m}$ were then patterned by electron beam lithography and ion beam etching. Finally, a MTJ consisting in a $\mathrm{Pt}(5 \mathrm{~nm}) / \mathrm{Fe}(15 \mathrm{~nm}) / \mathrm{MgO}(3.5 \mathrm{~nm})$ stack was grown at room temperature by electron beam evaporation and laterally defined by electron beam lithography. For the ISHE device (see Fig. 1b), starting from the same Ge surface, only a $15 \mathrm{~nm}$-thick Pt layer was grown on $\mathrm{Ge}$ at room temperature, with no $\mathrm{MgO}$ capping to allow electrons photogenerated in the $\mathrm{Ge}$ substrate to diffuse into the Pt ISHE detector. Eight Pt pads (identical to those fabricated on the MTJ device) and a $3 \times 1 \mu \mathrm{m}^{2} \mathrm{Pt}$ stripe for ISHE detection were then patterned by electron beam lithography and ion beam 

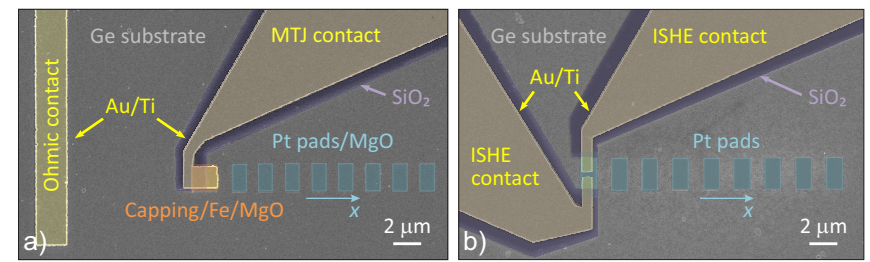

FIG. 1. (Color online) Scanning electron microscopy images of the lateral devices for optical spin generation and nonlocal electrical spin detection. For the MTJ device (a), the spininduced electrical signal is measured between the top layer and an ohmic contact directly grown on Ge, while the ISHE signal is measured (b) directly across the Pt stripe.

etching. Both MTJ and ISHE detectors where contacted by depositing an $\mathrm{Au}(250 \mathrm{~nm}) / \mathrm{Ti}(10 \mathrm{~nm})$ stack after passivating the surface with a $100 \mathrm{~nm}$-thick $\mathrm{SiO}_{2}$ layer. The magnetization of the MTJ layers and the position of the contacts on the Pt ISHE stripe are such that the measured signal in both detectors is sensitive only to the in-plane spin polarization in the $x$ direction of Figs. 1a and $1 \mathrm{~b}$.

A confocal scanning microscopy setup (Fig. 2a) provides maps of the $\Delta V$ signal from the spin detectors as a function of the position of a circularly-polarized light beam on the sample. Illumination is provided by a continuous wave laser diode working at a wavelength $\lambda=1550 \mathrm{~nm}(h \nu=0.8 \mathrm{eV})$, resonant with the direct band gap of Ge. The numerical aperture of the objective is 0.7 , giving a full-width at half maximum beam size of approximately $1.5 \mu \mathrm{m}$. The light is circularly polarized using the combination of a polarizer rotated at $45^{\circ}$ with respect to the axes of a photoelastic modulator. The circular polarization is modulated at $50 \mathrm{kHz}$, allowing for the synchronous detection of the electrical signal $\Delta V$ with a lock-in amplifier. Optical images were also simultaneously obtained by recording the reflected light from the sample with a near infrared InGaAs detector.

The generation of a spin-polarized electron population in the semiconductor occurs through the optical spin orientation process [7], consisting in the absorption of circularly-polarized light that generates spin-polarized electron-hole pairs at the $\Gamma$ point of the Brillouin zone. The spin polarization of photogenerated electrons in the conduction band is $P=\left(n_{\uparrow}-n_{\downarrow}\right) /\left(n_{\uparrow}+n_{\downarrow}\right)$, being $n_{\uparrow(\downarrow)}$ the spin-up (-down) densities referred to the quantization axis given by the direction of the light wavevector in the material. Photogenerated holes are rapidly depolarized due to their very short spin lifetime [20]. If the incident photon energy is tuned to the direct Ge bandgap, an electron spin polarization $P=50 \%$ can be achieved [27]. Right after the photogeneration, spin-oriented electrons thermalize from the $\Gamma$ to the $\mathrm{L}$ valleys within approximately $300 \mathrm{fs}$, maintaining most of their spin polarization [6].

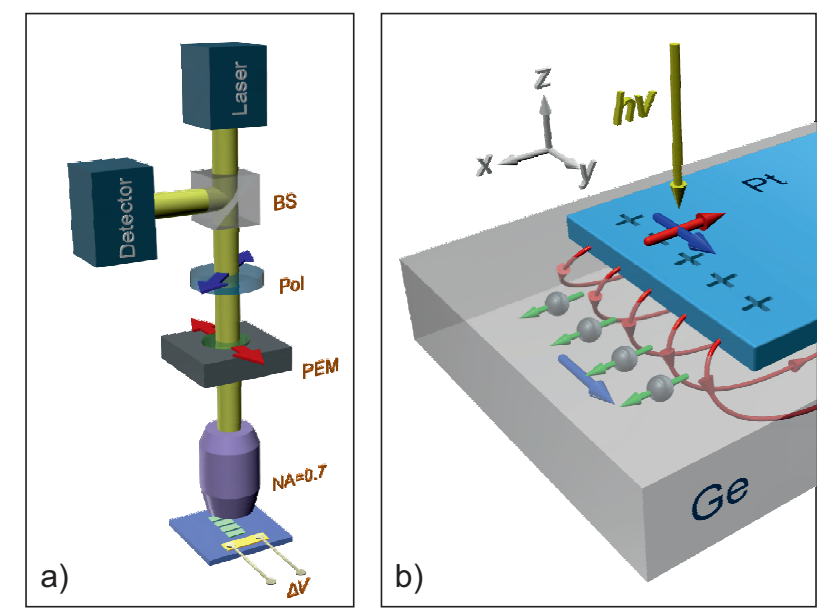

FIG. 2. (Color online) (a) Optical apparatus: $B S=$ beam splitter, $\mathrm{PEM}=$ photoelastic modulater, $\mathrm{Pol}=$ polarizer. $(\mathrm{b})$ Optical generation of in-plane spin-polarized electrons. When the light beam is focused below the edges of a $\mathrm{Pt}$ pad, the $x$ component (red arrow) of the illuminating field induces antiphase oscillating charges that, in turns, generate a staticlike near field (red field lines). Such a quasi-static field has a strong $z$ component that, combining with the $\pi / 2$ dephased $y$ component of the illuminating field (blue arrows), produces a circularly polarized electric field able to photoexcite in-plane spin-polarized electrons (green arrows). A complementary spin polarization is generated at the opposite edge.

At normal incidence on a uniform sample, only an out-of-plane spin polarization is generated, preventing any electrical spin detection in Ge with in-plane MTJ or ISHE detectors. The Pt pad pattern allows circumventing this limitation, as already demonstrated in Ref. 28. The physics of the process, which has been rigorously analyzed also by means of numerical simulations [28], is schematized in Fig. 2b: when the sample is illuminated with circularly-polarized light focused at the adge of a Pt pad, the $x$ component $E_{x}$ of the field (red arrow in Fig. 2b) induces charges that generate in the Ge substrate a near-field with a large component in the $z$ direction. The latter is in antiphase with respect to $E_{x}$ because the illumination wavelength of $1550 \mathrm{~nm}$ is significantly shorter than those corresponding to the main plasmonic resonances of the $\mathrm{Pt}$ pad. The combination of the $z$ component of the near field with the $\pi / 2$ phaseshifted $y$ component $E_{y}$ of the incoming light (blue arrows in Fig. 2b) results in an elliptic field polarization in the $y z$ plane, able to generate spin-polarized electrons along the $x$ axis. Opposite spin polarizations are attained at opposite edges of the Pt pads. The resulting spin accumulation creates a pure spin current (with no associated charge transport) detected nonlocally by either the MTJ or the ISHE detector. The same (not shown in Fig. 2b) also applies at correspondence with the edges perpendicular to the $y$ axis, where polarized photoelectrons are excited with spin oriented in the $y$ direction. However 

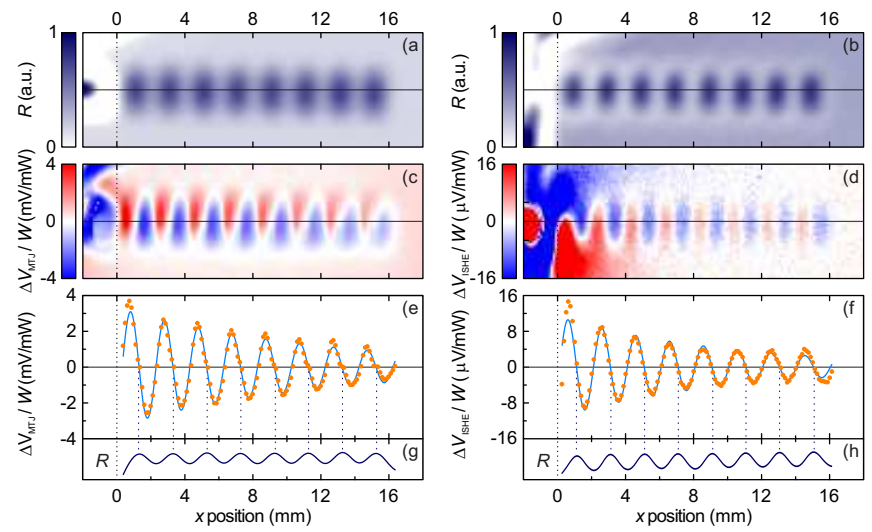

FIG. 3. (Color online) (a, b) Optical images recorded on the MTJ and ISHE devices, respectively. (c, d) Corresponding simultaneously recorded MTJ and ISHE voltage signals. (e, f) Voltage profiles along $x$ across the centers of the Pt pads. In both cases the detector is located at $x=0$. The dots are the experimental data while the solid lines correspond to fits (see text for the fitting function). The signals have been normalized to the laser power $W$. As illustrated by the dotted vertical line, corresponding to the maxima of the reflectivity profiles in (g) and (h), the signal is zero at the center of each Pt stripe and positive (negative) on the left (right) edge.

our spin MTJ- or ISHE-based detection schemes are not sensitive to this polarization axis and no signal is measured when the beam is focused on such edges [29].

The optical images of the nanostructures are shown in Fig. 3a (MTJ device) and 3b (ISHE device), while the room-temperature voltage signals are displayed in Fig. 3c for the MTJ (incident power $W=60 \mu \mathrm{W}$ ) and in Fig. 3d for the $\mathrm{Pt}$ ISHE detector (incident power $W=1.8 \mathrm{~mW}$ ), respectively. In Fig. 3e and 3f, the line profiles of the MTJ and ISHE voltage signals across the centers of the $\mathrm{Pt}$ pads are reported as a function of the distance to the detector. When the photon beam illuminates the Pt pad edges an alternating signal is observed, indicating opposite spin polarization injected at opposite edges. The amplitude of the oscillation decays when the beam is moved away from the detector as a consequence of the finite spin diffusion length in Ge. The possibility of varying the distance separating the spin injector from the detector allows for the measurement of the relative variation of the spin signal. Therefore, the fitting procedure can be performed using the spin diffusion length as the unique free parameter. In nonlocal experiments where the distance between the injector and the detector is fixed, the absolute spin signal variation must be fitted by means a suitable model which typically requires the knowledge of several physical quantities related to the efficiency of spin injection and detection $[14,15,21,30,31]$. As shown in Fig. 3e and 3f, the voltage $\Delta V$ normalized to the light power $W$ can be accurately fitted using an exponentially decaying sinusoidal function: $\Delta V / W \propto e^{-x / l_{s f}} \sin (2 \pi x / L)$, where $L=2 \mu \mathrm{m}$

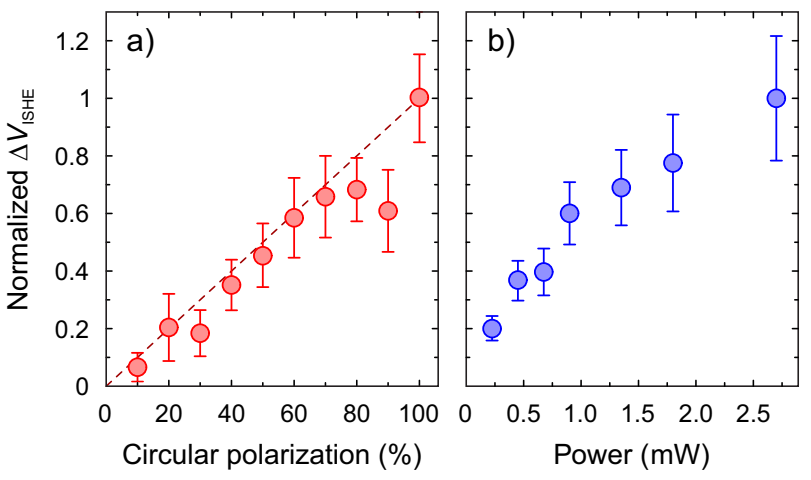

FIG. 4. (Color online) Dependence of the normalized $\Delta V_{\text {ISHE }}$ signal on the degree of circular polarization (a) and power (b) of the incident light. $\Delta V_{\text {ISHE }}$ is obtained from the interpolation shown in Fig. $3 \mathrm{f}$ and has been normalized to unity for a $100 \%$ circularly polarized beam with a power equal to $2.9 \mathrm{~mW}$. The degree of circular polarization has been varied by tuning the phase shift between the two components of the light electric field introduced by the PEM (see Fig. 2b)

is the pattern periodicity, $l_{s f}$ the spin diffusion length, and $x=0$ corresponds to the position of the detector.

By employing such a fitting expression, we implicitly assume a one-dimensional spin diffusion model, which is a rough approximation considering the three-dimensional geometry of our system. However, the very good agreement between the fitting curve and the experimental data suggests that the spin diffusion mostly takes place along $x$, which is probably due to the partial spin absorption by the Pt bars, which focus spin transport close to the Ge surface. We find: $l_{s f}=12 \pm 1 \mu \mathrm{m}$ for the MTJ device and $l_{s f}=10 \pm 1 \mu \mathrm{m}$ for the ISHE device. The difference between these values is related to the different sample architecture: in the MTJ device a $\mathrm{MgO}$ layer separates the Pt bars from the Ge substrate, whereas in the ISHE device $\mathrm{Pt}$ is directly in contact with Ge. Platinum acts as a spin sink: the presence of Pt bars between the generation and detection points reduces the number of spins reaching the detector. In the MTJ device, $\mathrm{MgO}$ partially prevents spin diffusion towards the $\mathrm{Pt}$ pads and the exponential decay can be mostly related to depolarization in the semiconductor. On the contrary, in the ISHE device, spin absorption in the $\mathrm{Pt}$ pads cannot be neglected and this reduces the effective spin diffusion length. Assuming an electron diffusion coefficient in the Ge substrate equal to $D=65 \mathrm{~cm}^{2} \mathrm{~s}^{-1}$ [32], in the $\mathrm{MgO}$-capped device we find a spin lifetime $\tau_{s f} \approx 20 \mathrm{~ns}$, which is longer than the theoretical estimation by Li et al. [4].

As a final remark, we would like to stress that the comparison between the MTJ and the ISHE detection clearly demonstrates the validity of the latter as a viable means to sample local spin currents. The combination of optical spin orientation with in-plane polarization and ISHE in a $\mathrm{Pt}$ bar thus defines an original nonlocal spin injection/detection scheme without the use of any ferromag- 
netic metal, which represents a new paradigm in the field of semiconductor spintronics. To further corroborate this conclusion, Figure 4 reports the dependence of the ISHE $\Delta V$ signal $\left(\Delta V_{\text {ISHE }}\right)$ measured across the detection $\mathrm{Pt}$ stripe as a function of the circular polarization degree and power of the incoming light. Indeed, the ISHE signal is proportional to the degree of circular polarization, hence to the photogenerated polarized spins in the material, while $\Delta V_{\text {ISHE }}$ tends to saturate at high illumination powers, as expected when a spin current is modulated by the photovoltage across a metal/semiconductor Schottky contact $[13,33]$.

In summary, we have demonstrated pure spin transport in Ge at room temperature using nonlocal optical spin orientation/electrical detection. We used either a MTJ or the inverse spin Hall effect in a Pt stripe to detect the in-plane spin signal generated in the Ge substrate by optical spin orientation at the edge of $\mathrm{Pt}$ nanostructures. The nonlocal evaluation of the spin accumulation as a function of the location where spin-polarized electrons are generated allows mapping spin diffusion paths inside the Ge substrate and evaluating characteristic diffusion lengths. The combination of locally-excited optical spin orientation with nonlocal ISHE-based spin detection represents a spintronic platform that is completely free from ferromagnetic functional units and from charge transport.

\section{ACKNOWLEDGEMENTS}

Financial support from the French National Research Agency through the ANR project SiGeSPIN \#ANR-13BS10-0002 and from the CARIPLO project SEARCHIV (grant 2013-0623) is acknowledged. The authors warmly acknowledge Paolo Biagioni for helpful discussion and Laurent Vila for assistance in the fabrication of the micro-devices.

* marco.finazzi@polimi.it

[1] D. D. Awschalom and M. E. Flatté, Nat.Phys. 3, 153 (2007).

[2] I. Žutić, J. Fabian, and S. Das Sarma, Rev. Mod. Phys. 76, 323 (2004).

[3] S. A. Wolf, D. D. Awschalom, R. A. Buhrman, J. M. Daughton, S. von Molnár, M. L. Roukes, A. Y. Chtchelkanova, and D. M. Treger, Science 294, 1488 (2001).

[4] P. Li, Y. Song, and H. Dery, Phys. Rev. B 86, 085202 (2012).

[5] F. Bottegoni, G. Isella, S. Cecchi, and F. Ciccacci, Appl. Phys. Lett. 98, 242107 (2011).

[6] F. Pezzoli, F. Bottegoni, D. Trivedi, F. Ciccacci, A. Giorgioni, P. Li, S. Cecchi, E. Grilli, Y. Song, M. Guzzi,
H. Dery, and G. Isella, Phys. Rev. Lett. 108, 156603 (2012).

[7] F. Meier and B. P. Zakharchenya, eds., Optical Orientation (North-Holland, 1984).

[8] E. J. Loren, J. Rioux, C. Lange, J. E. Sipe, H. M. van Driel, and A. L. Smirl, Phys. Rev. B 84, 214307 (2011).

[9] C. Guite and V. Venkataraman, Phys. Rev. Lett. 107, 166603 (2011).

[10] A. Giorgioni, E. Vitiello, E. Grilli, M. Guzzi, and F. Pezzoli, Applied Physics Letters 105, 152404 (2014).

[11] C. Rinaldi, M. Cantoni, D. Petti, A. Sottocorno, M. Leone, N. M. Caffrey, S. Sanvito, and R. Bertacco, Adv. Mater. 24, 3037 (2012).

[12] F. Bottegoni, A. Ferrari, S. Cecchi, M. Finazzi, F. Ciccacci, and G. Isella, Appl. Phys. Lett. 102, 152411 (2013).

[13] F. Bottegoni, A. Ferrari, F. Rortais, C. Vergnaud, A. Marty, G. Isella, M. Finazzi, M. Jamet, and F. Ciccacci, Phys. Rev. B 92, 214403 (2015).

[14] Y. Zhou, W. Han, L.-T. Chang, F. Xiu, M. Wang, M. Oehme, I. A. Fischer, J. Schulze, R. K. Kawakami, and K. L. Wang, Phys. Rev. B 84, 125323 (2011).

[15] L.-T. Chang, W. Han, Y. Zhou, J. Tang, I. A. Fischer, M. Oehme, J. Schulze, R. K. Kawakami, and K. L. Wang, Semicond. Sci. Technol. 28, 015018 (2013).

[16] E.-S. Liu, J. Nah, K. M. Varahramyan, and E. Tutuc, Nano Lett. 10, 3297 (2010).

[17] P. Li, J. Li, L. Qing, H. Dery, and I. Appelbaum, Phys. Rev. Lett. 111, 257204 (2013).

[18] A. Jain, L. Louahadj, J. Peiro, J. C. L. Breton, C. Vergnaud, A. Barski, C. Beigné, L. Notin, A. Marty, V. Baltz, S. Auffret, E. Augendre, H. Jaffrès, J. M. George, and M. Jamet, Applied Physics Letters 99, 162102 (2011).

[19] H. Saito, S. Watanabe, Y. Mineno, S. Sharma, R. Jansen, S. Yuasa, and K. Ando, Solid State Commun. 151, 1159 (2011).

[20] F. Rortais, S. Oyarzún, F. Bottegoni, J.-C. RojasSánchez, P. Laczkowski, A. Ferrari, C. Vergnaud, C. Ducruet, C. Beigné, N. Reyren, A. Marty, J.-P. Attané, L. Vila, S. Gambarelli, J. Widiez, F. Ciccacci, H. Jaffrès, J.-M. George, and M. Jamet, J. Phys.-Condes. Matter 28, 165801 (2016).

[21] A. Jain, J.-C. Rojas-Sanchez, M. Cubukcu, J. Peiro, J. C. Le Breton, E. Prestat, C. Vergnaud, L. Louahadj, C. Portemont, C. Ducruet, V. Baltz, A. Barski, P. Bayle-Guillemaud, L. Vila, J.-P. Attané, E. Augendre, G. Desfonds, S. Gambarelli, H. Jaffrès, J.-M. George, and M. Jamet, Phys. Rev. Lett. 109, 106603 (2012).

[22] A. Jain, C. Vergnaud, J. Peiro, J. C. L. Breton, E. Prestat, L. Louahadj, C. Portemont, C. Ducruet, V. Baltz, A. Marty, A. Barski, P. Bayle-Guillemaud, L. Vila, J.P. Attané, E. Augendre, H. Jaffrès, J.-M. George, and M. Jamet, Appl. Phys. Lett. 101, 022402 (2012).

[23] K.-R. Jeon, B.-C. Min, Y.-H. Jo, H.-S. Lee, I.-J. Shin, C.-Y. Park, S.-Y. Park, and S.-C. Shin, Phys. Rev. B 84, 165315 (2011).

[24] A. Hanbicki, S.-F. Cheng, R. Goswami, O. van't Erve, and B. Jonker, Solid State Commun. 152, 244 (2012).

[25] S. Iba, H. Saito, A. Spiesser, S. Watanabe, R. Jansen, S. Yuasa, and K. Ando, Appl. Phys. Express 5, 053004 (2012).

[26] S. Dushenko, M. Koike, Y. Ando, T. Shinjo, M. Myronov, and M. Shiraishi, Phys. Rev. Lett. 114, 196602 (2015). 
[27] J. Rioux and J. E. Sipe, Phys. Rev. B 81, 155215 (2010).

[28] F. Bottegoni, M. Celebrano, M. Bollani, P. Biagioni, G. Isella, F. Ciccacci, and M. Finazzi, Nat. Mater. 13, 790 (2014).

[29] It is worth noticing that MTJ and ISHE detectors have been designed to avoid spurious electrical effects related to the electron diffusion. At variance from Ref. 28, where the ISHE detection was performed with a continuous thin Pt film, with a bar-shaped ISHE detector we do not measure sizable signals related to a component of the spinpolarization parallel to the $\mathrm{Pt}$ pad edge. This indicates that the electromagnetic field modulation, operated by the Pt scatterers, mostly generates two complementary in-plane components of the spin polarization, perpendicular to the pad edges.

[30] K. Kasahara, Y. Fujita, S. Yamada, K. Sawano, M. Miyao, and K. Hamaya, Applied Physics Express 7, 033002 (2014).

[31] C. Rinaldi, S. Bertoli, M. Asa, L. Baldrati, C. Manzoni, M. Marangoni, G. Cerullo, M. Bianchi, R. Sordan, R. Bertacco, and M. Cantoni, Journal of Physics D: Applied Physics 49, 425104 (2016).

[32] A. L. Smirl, S. C. Moss, and J. R. Lindle, Phys. Rev. B 25, 2645 (1982).

[33] F. Bottegoni, C. Zucchetti, F. Ciccacci, M. Finazzi, and G. Isella, Applied Physics Letters 110, 042403 (2017). 